\title{
18 Kritische Anthropologie? - Zum Verhältnis zwischen Philosophischer Anthropologie und Frankfurter Kritischer Theorie
}

Auf einen ersten oberflächlichen Blick könnte es so scheinen, als stünde die Philosophische Anthropologie für die Hypothese eines von Natur aus unveränderlichen Wesens des Menschen und die Frankfurter Kritische Theorie für die Hypothese einer Revolution der Gesellschaft durch die Überwindung des Kapitalismus. Aber so einfach wie in diesem Gegensatz, der die wechselseitige Exklusion von Natur und künftiger Geschichte nur variiert, liegen die Dinge im Verhältnis beider philosophischer Richtungen nicht, da sich beide als dezidiert antidualistisch verstehen, ohne im Ganzen einen idealistisch oder materialistisch reduktiven Monismus zu vertreten. Für beide Richtungen ist Natur nicht allein ein unveränderlicher Determinismus, sondern auch eine Ermöglichungsstruktur geschichtlicher Veränderung, und die künftige Geschichtlichkeit gilt nicht einfach als eine idealistische Selbstbestätigung, sondern zugleich als ein materieller Prozess. Inwiefern die Wesensstruktur des Menschen als eine in sich widersprüchliche gerade geschichtliche Emanzipation ermöglicht und solche Emanzipation auf diese wesentliche, keineswegs beliebige Ermöglichungsstruktur angewiesen bleibt, ist eine lohnende Forschungsfrage, in die man durch das Verhältnis zwischen beiden philosophischen Richtungen hineingelangt. Zudem haben wir Heutige einen historischen Abstand zu den Begründern beider Richtungen, der die Freiheit zu einem systematischen Neuanfang gewährt. Auf diese Perspektive eines Neuanfangs verweist die Frage nach der Möglichkeit einer „kritischen Anthropologie“ (Herbert Schnädelbach), in der sich beide Richtungen ergänzen könnten. Auf dem Wege dorthin gilt es, einige ausgewählte problemgeschichtliche Stationen so zu durchlaufen, dass die wichtigsten systematischen Optionen aus beiden Richtungen im Verhältnis zueinander vor Augen stehen. Dabei werde ich mich auf Theodor W. Adorno, Max Horkheimer und Helmuth Plessner aus der Begründergeneration konzentrieren und durch die Stellungnamen von Jürgen Habermas in der Frage nach der Komplementarität beider Richtungen ankommen.

\section{1 „Kritische Theorie“ in welchem Sinne?}

Es dürfte unstrittig sein, bei der Beantwortung dieser Frage auf Max Horkheimers dafür kanonischen Text über Traditionelle und kritische Theorie einschließ- 
lich des Nachtrags (1937, hier 1988c u. 1988b) aus seinem Exil in New York zurückzugehen. Horkheimer lehnt „allgemeine Kriterien“ für den kritischen Charakter dieser Theorie ab (Horkheimer 1988c, 215). Gleichwohl ergebe sich ein gewisses Minimum an positiver Orientierung der Kritik. Eine gewisse Stabilität der Kritik folge zunächst aus ihrem Gegenstand der kapitalistischen Warenproduktion, die in ihrer nachliberalen Phase zu Monopolen geführt hat. Dadurch setze sich eine Verkürzung der Vernunft auf die Rationalisierung der Mittelwahl für angeblich vorgegebene Zwecke in der ganzen Gesellschaft durch, während es real um die Profitmaximierung für die „private Verfügungsgewalt über die Produktionsmittel, dieses Kernstück der herrschenden Gesellschaftsordnung“ (ebd., 209f.), gehe. Gegen diese monopolkapitalistische Entwicklung, deren Machtkonzentration sich in den „Verhältnissen der autoritären Staaten“ (ebd., 210) fortsetze, wird zunächst die klassisch bürgerliche Vernunftauffassung mit Kant und Hegel in Anschlag gebracht. Diese weite, vor allem praktische Vernunftkonzeption aus den revolutionären Zeiten des bürgerlichen Aufstiegs wird jedoch im Sinne von Marx transformiert. Die Ideale der Französischen Revolution werden auch für die Lösung sozialer Antagonismen in Stellung gebracht, woraus eine Orientierung vor allem an der Solidarität statt der Konkurrenz folgt. Während „traditionelle“ Theorien in ihrer arbeitsteilig verselbstständigten Funktion einen Beitrag zur Reproduktion der gegebenen Gesellschaft leisten, nehme die „kritische“ Theorie am „Kampf“ (ebd., 171, 181, 190) um die emanzipatorische Veränderung dieser Gesellschaft teil. Sie vertritt in diesem Sinne eine „Einheit von Theorie und Praxis“ gegen „die Hypostasierung des cartesianischen Dualismus von Denken und Sein“ (ebd., 205, siehe auch 185). Gegen die „Trennungen“ (ebd., 177) arbeitsteiliger Funktionen gegeneinander, die sich so dem Ganzen „entfremden“ (ebd., 182), werden die „dialektischen“ Einordnungen der Teile ins geschichtliche Ganze (Horkheimer 1988b, 224) zur Aufgabe gemacht. Dem entspricht ein interdisziplinäres Programm zur Sozialforschung, das die relevanten Einzelwissenschaften in die kritische Theorie der Gesellschaft integriert.

Da Horkheimer die Einheit von Gegensätzen, d. h. einen Widerspruch, für im Kapitalismus real gegeben hält, zeichnet er die künftige Gesellschaft durch „Identität“ aus: „Im Denken über den Menschen klaffen Subjekt und Objekt auseinander, ihre Identität liegt in der Zukunft und nicht in der Gegenwart“ (Horkheimer 1988c, 185). Horkheimer formuliert ausdrücklich das anthropologische Problem der kritischen Theorie, wenn er zwischen der gegenwärtigen kapitalistischen Entfremdung und der künftigen Identität von Individuum und Gesellschaft im Arbeitsprozess unterscheidet: 
Das kritische Denken enthält einen Begriff des Menschen, der sich selbst widerstreitet, solange diese Identität nicht hergestellt ist. Wenn von Vernunft bestimmtes Handeln zum Menschen gehört, ist die gegebene gesellschaftliche Praxis, welche das Dasein bis in die Einzelheiten formt, unmenschlich, und diese Unmenschlichkeit wirkt auf alles zurück, was sich in der Gesellschaft vollzieht. (Ebd., 183f.)

Der Widerstreit zwischen Gegenwart und Zukunft wird durch ein geschichtsphilosophisches Vor-Urteil überbrückt: Die „kritische Gesellschaftstheorie als ganze“ (ebd., 201) sei ein

einziges entfaltetes Existenzialurteil. Es besagt, grob formuliert, dass die Grundform der historisch gegebenen Warenwirtschaft, auf der die neuere Geschichte beruht, die inneren und äußeren Gegensätze der Epoche in sich schließt, in verschärfter Form stets aufs neue zeitigt und nach einer Periode des Aufstiegs, der Entfaltung menschlicher Kräfte, der Emanzipation des Individuums, nach einer ungeheuren Ausbreitung der menschlichen Macht über die Natur schließlich die weitere Entwicklung hemmt und die Menschheit einer neuen Barbarei zutreibt. (Ebd., siehe auch 208)

$\mathrm{Zu}$ der künftigen Identität passt, dass die „künftige Gesellschaft“ als die „Gemeinschaft freier Menschen" (ebd., 191) verstanden wird.

In der Organisation und Gemeinschaft der Kämpfenden erscheint trotz aller Disziplin, die in der Notwendigkeit, sich durchzusetzen, begründet ist, etwas von der Freiheit und Spontaneität der Zukunft. Wo die Einheit von Disziplin und Spontaneität verschwunden ist, verwandelt sich die Bewegung in eine Angelegenheit ihrer eigenen Bürokratie. (Ebd., 192)

Die Frage nach der Vergesellschaftung der wichtigsten Produktionsmittel wird nicht mit ihrer Verstaatlichung verwechselt, sondern ausdrücklich an die Entfaltung „realer Demokratie und Assoziation“ (Horkheimer 1988b, 223) gebunden.

Gegenüber diesem Minimum an positiver Orientierung in der Kritik hebt Horkheimer aber den Primat des Negativen in der Kritik hervor, da sich die künftige Gesellschaft nicht wie in einem Labor der traditionell arbeitsteiligen Theorien erzeugen lasse, sondern der Krise, Bewegung und Spontaneität im geschichtlichen Kampf bedürfe. Für das Negative in der Kritik steht bei ihm wiederholt die Forderung, Ausbeutung und Klassenherrschaft aufzuheben:

Diese negative Formulierung ist, auf einen abstrakten Ausdruck gebracht, der materialistische Inhalt des idealistischen Begriffs der Vernunft. In einer geschichtlichen Periode wie dieser ist die wahre Theorie nicht sosehr affirmativ als kritisch, wie auch das ihr gemäße Handeln nicht ,produktiv‘ sein kann. (Horkheimer 1988c, 216) 


\section{2 „Philosophische Anthropologie“ in welchem Sinne?}

Es dürfte ebenso unstrittig sein, zur Beantwortung dieser Frage auf Plessners Antrittsrede in seinem Groninger Exil über Die Aufgabe der Philosophischen Anthropologie aus demselben Jahre 1937 (hier 1983d) wie Horkheimers Aufsatz $\mathrm{zu}$ sprechen $\mathrm{zu}$ kommen, weil sich die Bezugnahme darauf seit Habermas (1958), Honneth und Joas (1980) und Schnädelbach (1983) zu Recht eingebürgert hat. Als philosophische Richtung (im Unterschied zur gleichnamigen philosophischen Subdisziplin) spricht die Philosophische Anthropologie erstens theoretisch den Menschen „als Menschen“ an: Dieser hermeneutische Anspruch verlässt sich weder auf eine zoologische „Spezies Homo sapiens“ noch beruht er auf der Exklusion Anderer durch die eigene Religion, Kultur oder das eigene Volk. „In der Philosophischen Anthropologie ist der Mensch als Mensch angesprochen und in diesem Zusatz eine Einschränkung auf den Bereich vor-genommen, der zwischen den Extremen größtmöglicher Vereinzelung und größtmöglicher Verallgemeinerung eine nicht genau festzulegende Mitte hält“ (Plessner 1983d, 36). Die wirkliche Mitte, in der sich die „Wesensverfassung“ zwischen Einzelnem und Allgemeinem im Menschsein vollzieht, wird selbst zur theoretischen Forschungsaufgabe, statt sie für entweder eine einzige Vereinzelung (die individuell je unvertretbare Existenz) oder eine einzige Verallgemeinerung (als Spezies, Gesellschaft, Kultur, Sprache, Vernunft) des Menschseins von vornherein auszuschließen.

Zweitens bedeutet der Zusatz ,als Mensch“ einen praktischen Anspruch, für dessen Erfüllung ebenso wenig allgemein anerkannte Garantien gegeben werden können. Dass wir Menschen sind und sein sollen, diese Entdeckung oder diese Forderung verdanken wir einer bestimmten Geschichte, der griechischen Antike und der jüdisch-christlichen Religiosität. (Ebd., 37)

Weil wir inzwischen aus historischer Erfahrung,

durch die Kritik der Entwicklungsidee, durch die politische und ideologische Bekämpfbarkeit der humanitas um die Gewagtheit und Rückhaltlosigkeit des ,Menschen“Gedankens wissen, müssen wir das Menschsein in der denkbar größten Fülle an Möglichkeiten, in seiner unbeherrschbaren Vieldeutigkeit und realen Gefährdetheit so zum Ansatz bringen, dass die Gewagtheit eines derartigen Begriffes als Übernahme einer besonderen Verantwortung vor der Geschichte verständlich wird. (Ebd.)

Die Philosophische Anthropologie geht diese theoretisch-praktische Aufgabe an, indem sie drei Grundsätzen im Hinblick auf eine dreifache Verbindung folgt, eine Verbindung nicht nur zur Philosophie, sondern auch zu den Einzelwissenschaften und zur geschichtlichen Situation. Erstens gelte in der Philoso- 
phischen Anthropologie „die methodische Gleichwertigkeit aller Aspekte, in denen menschliches Sein und Tun sich offenbart“, darunter des physischen, psychischen, geistig-sittlichen und religiösen Aspekts, „für die sogenannte Wesenserkenntnis vom Menschen“ (Plessner 1983d, 39). Diese methodische Gleichwertigkeit richtet sich gegen alle „materialistischen, idealistischen, existentialistischen Einseitigkeiten“ (ebd.). Zweitens gehe es um die Art und Weise der Einheit dieser Aspekte und damit des Überganges zwischen ihnen. Diese Art und Weise von Einheit sei von derselben „Ursprünglichkeit, wie sie der Mensch in seinem Geschichte werdenden Dasein beweist, in dem er sie sich erringt“" (ebd.). Es handelt sich also um eine qualitative Einheit der Aspekte, die in dem phänomenologischen Sinne „ursprünglich“ ist, dass sie nicht aus der Erkenntnis anderer Sachverhalte abgeleitet werden kann. Sie ist lebensweltlich durch keine „isolierende Methode des Laboratoriums“ (ebd., 47) zur Darstellung und Messung einzelwissenschaftlicher Erkenntnisse zu ersetzen. Diese qualitative Einheit ist aber auch nicht unbefragbar vorgegeben, sondern aus dem in der Geschichte werdenden Dasein errungen, also hermeneutisch zu erschließen. Ihre Veränderung kann besser oder schlechter ausfallen, was zu beurteilen eine „positive Zusammenarbeit“ mit den „Natur- und Kulturwissenschaften vom Menschen“ erfordert: Plessner gibt das Beispiel von der Erkenntnis „der Wirkungseinheit der Person zwischen Leib und Körper, Körper und Geist“ im biomedizinischen Kontext zwischen Arzt und Patient, wodurch auch die „Erkenntnistheorie und Ontologie vor neue Aufgaben“ gestellt werden (ebd., 48). Der dritte Grundsatz bezieht sich auf die geschichtliche Situation, in der die überweltlichen Autoritäten zerfallen sind und die innerweltlichen Autoritäten angesichts von Individualisierung und Pluralisierung strittig bleiben (ebd., $41 \mathrm{mit}$ Verweis auf Plessner 1974). Er „,bestimmt die Funktion der Philosophischen Anthropologie, die sich ihrer theoretischen Grenzen im Hinblick auf ihre praktische Verantwortung gegen die Unergründlichkeit des Menschenmöglichen bewusst ist“ (Plessner 1983d, 39; 44 mit Verweis auf Plessner 1981d). Daher können die Strukturformeln für die Erkenntnis der Wesensverfassung „keinen abschließend-theoretischen, sondern nur einen aufschließend-exponierenden Wert" beanspruchen: In dieser Sicherung der Unergründlichkeit des Menschen komme „der Ernst der Verantwortung vor allen Möglichkeiten“ zum Ausdruck, in denen sich der Mensch ,verstehen und also sein kann“ (Plessner 1983d, 39).

Nach der phänomenologischen und hermeneutischen Methode bringt Plessner auch eine bestimmte Art und Weise von Dialektik zur Sprache, die zweifellos auf Hegels Phänomenologie des Geistes anspielt, ohne Hegels systematisch positive Fassung des Absoluten als Geist Gottes zu beanspruchen. Die moderne Skepsis gegen jede Autorität rechne mit einer Selbsttäuschung, die 
durch ihre reflexive Rückführung auf ein bestimmtes Subjekt behoben werden könne, was sich von Kant über Marx und Nietzsche bis Freud oft genug wiederholt hat. Diese Skepsis sei auch gegen die „Selbstvergötterung des Menschen“ im Namen der „Fortschritte“ an „Verfügungsgewalt“ durch Wissenschaft und Technik zu richten (Plessner 1983d, 50), womit die Kritik immanent einsetzt. Aber die Verwirklichung dieser Skepsis überwinde sie auch, denn sie bringe Anderes zum Vorschein, als in der dualistischen Art von Skepsis an alleiniger Selbsttäuschung eines Subjekts erwartet werde. Die Verwirklichung dieser Skepsis lasse die „Grenze“ klar werden, „bis zu der sich der Mensch als Mensch in Frage stellen kann“ (ebd., 45): Ohne ihre Verwirklichung wird man „den Verdacht gegen das Recht, vom Menschen als einem besonderen und auf seinen Gattungscharakter verpflichteten Wesen zu sprechen, nicht loswerden“ (ebd.). Der durch seine „Destruktion“ führende Begriff des „Menschen“ habe „Wagnischarakter“ und erfordere den „Mut zur rückhaltlosen Skepsis als einer Methode des Menschen, sich durch Selbstentsicherung wiederzufinden“ (ebd., 46). So heißt es denn zusammenfassend: „Die im Sinne ihrer Überwindung verwirklichte Skepsis ist allein möglich als Philosophische Anthropologie“ (ebd., 41, Hervorhebung im Original).

\subsection{Zu Horkheimers und Adornos Kritiken an Philosophischen Anthropologien}

Es gibt zahlreiche Kritiken von Adorno und Horkheimer an naturalistisch reduktiven Anthropologien und an philosophisch pessimistischen oder optimistischen Anthropologien in der Tradition von Hobbes und Rousseau. Im Kern bedeutet ihre Kritik immer, dem oben angesprochenen Verständnis von Gesellschaftsveränderung gemäß, diese Anthropologien oder Menschenbilder - oft wird beides nicht unterschieden - als eine Verewigung kapitalistischer Verhältnisse im Namen der Natur zu demaskieren, analog zu Marx' Kritik an bürgerlicher Ideologie und Wissenschaft. Schwieriger war es bereits, die philosophischen Anthropologien Ludwig Feuerbachs und des jungen Marx in ein geschichtsphilosophisches Nacheinander von kapitalistischer und künftiger Gesellschaft zu drehen. Unter den kritisierten zeitgenössischen philosophischen Anthropologien befinden sich die verschiedenen Zugänge von Max Scheler und Arnold Gehlen. Bei aller Bewunderung Horkheimers für Scheler, dessen philosophisch souveränen Umgang mit einem großen Reichtum einzelwissenschaftlicher Erkenntnisse, seine Wissenssoziologie und seine antikapitalistische Sinnordnung von Mitgefühlen, werden an Schelers philosophischer Anthropologie vor allem ihre seinsmetaphysisch-spekulative Einbindung und ihre Unterschät- 
zung der Klassenspaltung zugunsten der Einheit des Menschen kritisiert (siehe Horkheimer 1987 u. 1988c). An Gehlens philosophischer Anthropologie werden von Adorno ihre positivistischen und autoritären Charakteristika kritisch herausgestellt, nicht ohne ihren zeitdiagnostischen und konservativ polemischen Charakter zu schätzen, der es ihm ermöglichte, die eigene linke Kritik in Szene zu setzen, ohne die kulturkritischen Gemeinsamkeiten thematisieren zu müssen (siehe Thies 1997).

Auffallend ist indessen, dass es keine Kritik von Horkheimer oder Adorno an namentlich Plessners Philosophischer Anthropologie gibt. Sie schweigen sich zu ihr öffentlich aus, wodurch unklar bleibt, ob sie erkannt haben, warum sie diese Philosophische Anthropologie im Unterschied zu der von Scheler und Gehlen nicht kritisieren können oder warum sie sogar dieser und jener ihrer Dimensionen folgen könnten. Offenbar setzte Habermas ab 1958 das Niveau und den Standard in der Verhältnisbestimmung beider Richtungen. Adorno und Horkheimer teilten mit Plessner die Problematik der Emigration und Remigration. Sie vertrauten einander politisch und kollegial, wovon Plessners Vertretung von Adorno 1952/53 in Frankfurt sowie ihr Briefwechsel zeugt und wofür gemeinsame Familienurlaube sprechen (siehe Wiggershaus 1986, 511-514; Monika Plessner 1995). Bekannt ist auch die wissenschaftspolitisch strategische $\mathrm{Zu}$ sammenarbeit der Frankfurter mit Plessner in Fragen der empirischen und theoretischen Sozialforschung (siehe Dietze 2006, 370, 445f., 516; Fischer 2008, 278f., 289, 311-314), aber sie scheint weder in der einen noch in der anderen Richtung wirklich philosophische Konsequenzen gehabt zu haben. Schaut man in Horkheimers und Adornos Beiträge zu der Festschrift für Helmuth Plessner Wesen und Wirklichkeit des Menschen (1957), wird deutlich, dass es zu keiner philosophischen Auseinandersetzung über ihre Paradigmen gekommen ist. Adorno handelt dort von Soziologie und empirischer Forschung, Horkheimer geht auf Kant und Hegel zurück, um dann zeitdiagnostisch zum sozialhistorischen Wandel von Menschenbildern Ausführungen zu machen (siehe Adorno 1957; Horkheimer 1957 in Ziegler 1957).

Die einzige Stelle bei Adorno, die indirekt außer Schelers auch Plessners Philosophische Anthropologie meinen könnte, betrifft das in Frankfurter Kreisen berühmte Diktum Adornos gegen jegliche Anthropologie. Das Primat des Negativen über das Positive im kritischen Charakter der Kritischen Theorie hat philosophisch gesehen sicherlich Adorno am besten in Negative Dialektik (1966) ausgearbeitet. Durch seine Negativität zur Verteidigung des Nicht-Identischen im Singulären gegen dessen begriffliche Identifikationen wurde auch Horkheimers identitätsphilosophische Antizipation der künftigen Gesellschaft als eine Gemeinschaft (1937, hier 1988c) obsolet. Für Adorno aber bleibt diese 
Negativität das Vorrecht seiner dialektischen Philosophie, während der Ausdruck „Anthropologie“ auf ideologische Menschenbilder bezogen wird. Daher reagiert Adorno besonders gereizt auf die These einer ,arrivierten Anthropologie“, d. h. einer Anthropologie, die nicht in sein Begriffsschema passt, und die besagt, der Mensch sei offen, was Adorno als eine leere These abtut. „Dass nicht sich sagen lässt, was der Mensch sei, ist keine besonders erhabene Anthropologie, sondern ein Veto gegen jegliche“ (Adorno 1966, 128). ${ }^{1}$ Gerhard Gamm kommentiert diesen Satz Adornos treffend, wenn er schreibt:

Adornos Einwand, sollte er denn gegen die philosophische Anthropologie Plessners gemünzt sein, ist selbst kein besonders erhabener. Eine Dialektik, die aufs Nicht-Identische, auf die ,Nicht-Identität des Menschen mit sich', abhebt, bedarf notwendig jenes Moments der ,Unbestimmtheitsrelation des Menschen zu sich', von der Plessner spricht. (Gamm 2015, 151; siehe auch schon Gamm 2006)

Schaut man sich den Kontext dieses Adorno-Zitats an, ist klar, dass es sich um eine Kritik an Heideggers Humanismusbrief handelt. Diesem zufolge steht der Mensch in die „Offenheit des Seins“ aus und wird daher unter seiner Würde gedacht, wenn er als „Lebewesen“ thematisiert wird, obgleich man die Dürftigkeit seines Lebens ernst zu nehmen habe, aber aus der Lichtung des Seins heraus (siehe Heidegger 1967, 320, 346, 352). Gleichwohl fragt sich, warum dann Adorno Heideggers Anspruch auf eine „Fundamentalontologie“ kritisch eine „Anthropologie“ nennt. - Dies folgt für Adorno aus Heideggers eigener Behauptung, die Adorno zitiert: „Der Satz: ,Der Mensch eksistiert“ antwortet nicht auf die Frage, ob der Mensch wirklich sei oder nicht, sondern antwortet auf die Frage nach dem ,Wesen“ des Menschen“ (Heidegger 1954, 70f.; zitiert in Adorno 1966, 121f.). Aber warum erweitert dann Adorno seine Kritik an Heideggers Anthropologie auf ,jegliche“ Anthropologie? Hierdurch wird Schelers und Plessners These von der Weltoffenheit personalen Lebens, die Heidegger zweifellos in der offenen Lichtung des Seins übertrumpfen wollte (siehe Krüger 2001, 128-143), in die Kritik einbezogen. Heidegger und Plessner gingen beide von Schelers ekstatischer Eröffnung von Welt in geistiger Liebe aus, transformierten sie aber in entgegengesetzte Richtungen (siehe Krüger 2012, 18-23; im vorliegenden Band 23. Kapitel). In gewisser Weise kommt der späte Heidegger wieder in Schelers seinsspekulativer „Durchdringung“ von Lebensdrang und Negativität des Geistes (Scheler 1995, 87) an, allerdings nun auf einer Lichtung ohne Humanität, d. h. in einer Adorno zu Recht perfiden Leerheit, in der sich welthis-

1 Schüßler versteht diese Stelle als Plessner-Kritik (Schüßler 2000, 184, vgl. insgesamt ebd., 180-186). 
torisch gerade die größten Zivilisationsverbrechen ereignet hatten. Während sich aber Karl Löwith differenziert für Plessners Weg der „Überschreitung“ in die „Natur selber“ (Löwith 1957, 85) ${ }^{2}$ gegen Heideggers Weg in ein leeres Sein als Maske jeder möglichen Selbstermächtigung positionierte, reagierte Adorno auf diese gesamte Problemkonstellation nicht nur spät, sondern bekenntnishaft. Umso wichtiger wäre die differenzierte Befragung des Zusammenhangs zwischen einer ,negativen Anthropologie“ (Gamm mit Plessner) und einer ,negativen Dialektik“ (Adorno), den in der Gegenwart Christoph Menke (2008) als ästhetische Anthropologie entwickelt.

\subsection{Plessners Kritiken am Marxismus (1960 und 1963)}

Sieht man umgekehrt in Plessners Beitrag Immer noch Philosophische Anthropologie? (hier Plessner 1983i) zu von Horkheimer herausgegebenen Festschrift für Adorno Zeugnisse (1963), so kommt Plessners Erläuterung seiner Frageweise nach dem Menschen der Kritischen Theorie dadurch entgegen, dass sie geschichtlich erfolgt. Er wiederholt hier in äußerst knapper Form seine Kritik am dualistischen Mainstream der modernen Philosophie, wie er sie bereits 1935 in seinem Buch Das Schicksal deutschen Geistes im Ausgang seiner bürgerlichen Epoche vorgetragen hatte, das ab 1959 unter dem Titel Die verspätete Nation (hier Plessner 1974) erneut publiziert worden war. Seit Descartes habe man, mit Kant gesprochen, immer wieder eine kopernikanische Revolution vom Standpunkt eines Ex-Zentrums aus versprochen. Im Ergebnis sei man aber immer wieder durch dualistische Abspaltungen von der Welt der Körper und Leiber, von der Natur im Ganzen, in einem Subjekt qua Selbst gelandet, das sich für den Konstitutionsgrund der Welt halte. So habe z. B. Dilthey „Kants kopernikanische Wendung noch einmal auf sie angewendet, ohne in die verlassene ontologische Konzeption“ von Welt zurück zu schwenken (Plessner 1983i, 242). ${ }^{3}$ Jede

2 Löwith (1957), 85: „Das Überschreiten, welches den Menschen und seine Sprache vom Tier unterscheidet, könnte sich noch immer im unüberschreitbaren Umkreis der Natur vollziehen, ohne Ausgriff nach einem Jenseits des von Natur aus Seienden, so dass der Terminus a quo und ad quem des menschlichen Überschreitens ein und derselbe, die Natur selber wäre.“

3 Bereits 1935 hatte Plessner über den Ausgang des Überbietungskampfs an gegenseitigen Entlarvungen geschrieben: „Die überweltliche Heilsordnung weicht der Vernunft, diese der Geschichte, diese der Ökonomie und Gesellschaft, und ihre Stelle nimmt schließlich das Blut ein. Für die himmlische Verheißung und Erlösung gibt es die innerweltliche Verheißung und Erlösung in einer Evolution des Wissens und Könnens, und als es mit dem Glauben an den 
Bewegung verspricht eine Emanzipation, indem sie die Anderen von ihrem Exzentrum aus dezentriert, das sich sodann aber nur als ein je eigener Existenzanspruch in Konkurrenz zu anderen entpuppt. „Die Relativierung des Bewusstseins auf gesellschaftliche und vitale Triebkräfte beendet eine Geschichte der Emanzipation des Menschen von der Welt, die in der Philosophie von Descartes bis zum Existentialismus aufgezeichnet ist“ (ebd., 235).

Dies ist ein hartes Urteil, das die oben erwähnte Skepsis wider die Autorität durch Rückführung auf ein Subjekt erneut aufruft, dessen geschichtliche Pluralisierung im Bürgerkrieg untergeht. Die Emanzipation eines Subjekts erweise sich als seine Emanzipation von der Welt, die dann geschichtlich überraschend in der Gestalt gesellschaftlicher und vitaler Triebkräfte über die Subjekte hereinbricht. Damit entsteht das Problem, wie denn diese Skepsisart in ihrer Verwirklichung überwunden werden kann, erneut. Plessners Diagnose ähnelt der von Hannah Arendt aus The Human Condition (1958): Das Grundproblem bestehe seit der Neuzeit nicht darin, dass wir die Entfremdung von unserem je eigenen, individuellen oder kollektiven Selbst überwinden müssten, wie gemeinhin geglaubt werde, sondern darin, dass wir keine Welt mehr teilten, die uns miteinander und gegeneinander ermöglichen könnte (siehe Arendt 1981b, 265, 325, 383, 408).

Plessner geht den dualistischen Umschlag des Emanzipationsversprechens in die Ausklammerung der Natur, d. h. der Welt der Körper und Leiber und ihrer Relationen, für alle drei Methoden seines Projektes kurz durch, für die Phänomenologie, die Hermeneutik und die Dialektik. Alle drei versprechen radikal Neues und endliche Emanzipation, landen im dualistischen Ergebnis aber in der Vergrößerung der Kluft zwischen Subjekt und Welt. So feindlich sich darunter die Richtungen des Marxismus und des Existenzialismus gegenüberstünden, weil für sie alles von dem Gegensatz zwischen kollektivem und individuellem Selbst abhängen soll, seien sie sich doch einig im Kampf gegen die Entfremdung nicht etwa von der Welt sondern von ihrer jeweiligen kollektiven oder individuellen Existenz als Maßstab für die Zukunft. Selbst von Ernst Cassirers symbolischen Formen über Heideggers sprachliches Haus des Seins bis zur linguistic analysis kenne man in all diesen „anthropologischen Philosophien“ (Plessner 1983i, 243) schon immer das Wesen des Menschen. Es liege für sie in der Sprache der Symbole, zwischen Sprachverklärung und Sprachverarmung, ohne Relevanz für die Phänomene der Welt, wie sie sich selber in ihr zeigen können. Immer bleibe dualistisch gedacht die Natur, d. h. die Welt der Körper

Fortschritt schließlich zu Ende ist, die vor- und untermenschliche Sicherung des Menschen im Volkstum“ (Plessner 1974, 119). 
und Leiber, das Andere und Fremde, das für die Leistungen von Subjekten zu funktionieren habe, das man mithin nicht als Selbst und Welt sein lassen kön$n e .{ }^{4}$ Für die „Überwindung des Anthropozentrismus“ nimmt Plessners Philosophische Anthropologie gerade die dualistisch ausgeschlossene Natur, diese vom Dualismus exkludierte Welt der Körper und Leiber, in Anspruch, weil er sie phänomenologisch, hermeneutisch und dialektisch als Ermöglichungsstruktur des Geistigen inkludiert. Die Natur exzentriert sich in eine „Sphäre des Menschen“ (Plessner 1975, 288), nicht aber dieses Individuum, jene Rasse oder Klasse dezentriert sich selbst gegen alle anderen: „Wir auf der Erde kennen den Menschen nur als Hominiden. Wer aber sagt uns, dass seine Lebensgestalt die einzig mögliche für ein endliches Wesen ist, das über Einsicht und schöpferische Kraft verfügt" (Plessner 1983i, 246f.)? Plessner erinnert in seinem Beitrag zu der Festschrift für Adorno 1963 daran, dass seine „Philosophische Anthropologie“ seit 1937 expressis verbis gegen ,anthropologische Philosophien“ entworfen worden ist, die vermeintlich um das Wesen des Menschen bereits wissen und auf dieser Grundlage philosophieren zu können meinen (ebd., 243; siehe schon Plessner 1983d, 36). Merkwürdigerweise ist seine Gegenüberstellung zwischen „Philosophischer Anthropologie“ und „anthropologischer Philosophie“ nicht rezipiert worden, auch nicht in den Überblicksdarstellungen von Habermas (1958) über Honneth und Joas (1980) bis Schnädelbach (1983) und Fischer (2008), obgleich sie viele Missverständnisse hätte vermeiden können (siehe Krüger 2009a; Wunsch 2014).

Fragt man sich, wie das Problem der gemeinsamen Entfremdung von der Welt statt von der je eigenen individuellen oder kollektiven Existenz angegangen werden kann, begibt man sich mit Plessner zunächst auf den Weg der „Mitwelt“ (im Unterschied zur Innen- und Außenwelt; dazu Plessner 1975, 293308), die politisch nur im „Öffentlichen“ (im Unterschied zum Privaten; dazu Plessner 1981b, 95) ${ }^{5}$ entstehen kann. Bereits 1960 war sein engagierter Aufsatz Das Problem der Öffentlichkeit und die Idee der Entfremdung erschienen: Wäh-

\footnotetext{
41931 hatte Plessner geschrieben (Plessner 1981d, 163-164): „Eine neue Verantwortung ist dem Menschen zugefallen, nachdem ihm die Durchrelativierung seiner geistigen Welt den Rekurs auf ein Absolutes wissensmäßig abgeschnitten hat: das Wirkliche gerade in seiner Relativierbarkeit als trotzdem Wirkliches sein zu lassen.“

5 In Plessner 1981b, 95 heißt es dazu: Öffentlichkeit ist „das offene System des Verkehrs zwischen unverbundenen Menschen“. Sie sind unverbunden, weil nicht im Sinne einer familienoder sachähnlichen Gemeinschaft verbunden. In diesem Verkehr müssen daher „künstliche Normen" generiert werden (ebd., 96), die sich gegen asymmetrische Gewalt und Willkür erst nach dem „Prinzip der Gegenseitigkeit“ stabilisieren lassen (ebd., 101) und unter modernen Bedingungen den Funktionswert des Schutzes der Menschenwürde annehmen (ebd., 102-103).
} 
rend der „spätbürgerliche Existentialismus“ die „Öffentlichkeit zur verflachten, uneigentlichen Weise menschlichen Daseins“ entwerte, „indem er Innerlichkeit mit Eigentlichkeit gleichsetzt“, entwerte der „proletarische Marxismus“ (Plessner 1985b, 225) die Öffentlichkeit

\begin{abstract}
in ihrer heutigen Form als Ausdruck des seiner Entfremdung noch nicht Herr gewordenen Menschen. Nichts ist in unserer Lage verhängnisvoller, als den Tröstungen zu vertrauen, welche die Idee der Entfremdung verspricht. Freiheit und Eigentlichkeit im Inneren zu suchen - der Appell des Existentialismus - fördert die Verdinglichung des Menschen in der Öffentlichkeit nicht weniger als die marxistische Eschatologie, die ihn in den Wartestand versetzt, im Blick auf das Ende der Geschichte. Nur ein gegen die Verführungen des Entfremdungsgedankens gefeiter, nur ein entmythisierter Öffentlichkeitsbegriff sichert den Spielraum der Verantwortung zur Wahrung unserer gesellschaftlichen Freiheit. (Ebd.)
\end{abstract}

Verwechsele man nicht die zweifellos elende Lage des Proletariats im 19. Jahrhundert mit der Lage nach der Durchsetzung „sozialer Bürgerrechte“, müsse man sowohl den „Prozess der Vergesellschaftung des Staates“, d. h. das „Ineinanderwachsen der früher getrennten Bereiche der Verwaltung, des Militärs, der Wirtschaft und Kultur“, als auch den über den Rechtsstaat hinausführenden „Wohlfahrtstaat“ (ebd., 215) ernst nehmen: „Vermassung besagt eine Relation der Unschärfe in der Abgrenzung beider Bereiche [des Öffentlichen und Privaten: HPK], die mit der Durchsetzung des sozialen Bürgerrechts in Bewegung geraten sind“ (ebd., 216). In dieser Lage plädiert Plessner für eine Entfaltung der Personalität zwischen einander je privaten und doch öffentlichen Doppelgängern. „Der Abstand, den die Rolle schafft, im Leben der Familie wie in dem der Berufe, der Arbeit, der Ämter, ist der den Menschen auszeichnende Umweg zum Mitmenschen, das Mittel seiner Unmittelbarkeit“ (ebd., 223f.) Man komme zu sich, als einem privaten Selbstverhältnis, auf dem Umweg über Andere im öffentlichen Austausch mit ihnen dank einer Rolle: Nicht Tiere, nicht Engel, aber „der Mensch erscheint als Doppelgänger, nach außen in der Figur seiner Rolle und nach innen, privat, als er selbst“ (ebd., 224).

\title{
18.5 Der späte Plessner zum späten Adorno: Die Brücke einer gemeinsamen anti-dualistischen Lektüre von Kants agnostischer Absolutismus-Kritik
}

Plessner stimmt in seiner Anthropologie der Sinne (1970, hier 1980a) Adorno darin zu, dass man Musik aus dem Musizieren heraus verstehen sollte, statt sie aus den sensorischen und motorischen Verhaltensmöglichkeiten herauszulösen und in das Gefängnis eines verselbstständigten Bewusstseins sperren zu müs- 
sen (Plessner 1980a, 356). Die Verzweiflung indessen, die in Adornos negativer Dialektik herrscht, teilt Plessner in seiner Besprechung dieses Buchs nicht. Plessner hing nicht der marxistischen Überzeugung an, man könne durch eine einzige Radikallösung, die Revolution, alle Probleme der modernen Gesellschaften wie durch ein „Allheilmittel“ überwinden. Seine Schrift Grenzen der Gemeinschaft. Zur Kritik des sozialen Radikalismus (1924, hier 1981b) enthielt eine grundsätzliche Kritik an der marxistischen Vorstellung, die kapitalistische Gesellschaft durch eine Rationalisierung nach dem Modell einer Sachgemeinschaft ersetzen zu können. Plessner hatte schon damals gegen jede Art von Gemeinschafts-Diktatur eine zivilisierte ${ }^{6}$ und öffentliche Gesellschaft unter einander Unbekannten, Anderen und Fremden stark gemacht, die einander unbekannt, anders und fremd bleiben können. Andererseits hat Plessner aber oft an marxistische Analysen, so die des „Hochkapitalismus“ (Plessner 1974, 87-89) 1935 in seinem Buch Das Schicksal deutschen Geistes im Ausgang seiner bürgerlichen Epoche angeschlossen. Er teilt auch jetzt, 1970, die Diagnose von der verwalteten Welt in West und Ost, aber ohne an ihr zu verzweifeln, weil es zu keiner revolutionären Erlösung von ihr komme: „Die Verknüpfung des Schlussstrichs unter die Hegelsche Philosophie mit dem Gedankenstrich der versäumten Revolution bildet das Scharnier der negativen Dialektik“ (Plessner 2001a, 266).

Lasse man Adornos marxistisches Epigonentum und seinen Mangel an systematischem Darstellungsvermögen beiseite, trete seine Stärke hervor: „Ein Philosoph muss eben auch ein Écrivain sein. Diese Gabe besaß Adorno, jedenfalls als Interpret großer Musik und Dichtung“ (Plessner 2001a, 271). Aktuell und interessant sei Adornos Versuch, durch ein „Ensemble von Modellanalysen“ der identitätsphilosophischen „Vormacht des Subjekts“ zu entkommen und der „Dignität des Körperlichen“ (ebd., 273-274) ohne dualistische Zwangsjacke nachzugehen. Adornos „Konstellierung“ der Begriffe, statt ihrer exklusiven Subsumtionshierarchie, und seine Komponierung historisch verstehender Typen (im Sinne Max Webers) werden von Plessner als Schritte in Richtung auf die geschichtliche Wirklichkeit hervorgehoben. Adornos Ausrichtung seiner Mikrologie auf die Materialität des Gehalts lasse zu Recht das Ontische nicht in seiner Ontologie aufgehen. „Alles in seiner Einzigkeit und Unwiederholbarkeit Unverwechselbare, ob sensuell oder nicht, ist das Thema der Metaphysik im

6 Dort (Plessner1981b, 93) verteidigte er die Zivilisation vor der angeblich höheren Kultur: „Mithin kann man sagen, gäbe es keine Zivilisation schon aus den einfachsten Zweckmäßigkeitsgründen, so müsste sie um des Spieltriebes willen erfunden werden [...]. Was aber ist der Sinn des Spieles, wenn nicht die Irrealisierung des natürlichen Menschen zur Trägerschaft irgendeiner Bedeutung, irgendeiner Rolle?“ 
Augenblick ihres Sturzes“ (ebd., 280). Literarisch laufe dies auf die Form des Essays hinaus, dem Franzosen und Engländer großen Respekt erwiesen, kaum aber die Deutschen (Plessner 2001e, 334).

Plessner erkennt in Adornos anti-dualistischer Kant-Lektüre eine Brücke zu seinem eigenen Projekt, weil sie sich vom positiven Absoluten der Identitätsphilosophie befreit. Die „Anerkennung der Materialität gesellschaftlicher Macht und das unbrechbare Reduit der sich selber bestimmenden Freiheit, Sinnlichkeit und Sittlichkeit“ gehören zusammen, ohne sich im Namen des ,absoluten Idealismus“ selbst ermächtigen zu können: „Das ist in einer Welt, der die Prinzipien der Aufklärung selber zu Mitteln in der Ideologisierung geworden sind und deren Fanatismus sie mit ihrer Selbstvernichtung bedroht, ein dringend nötiges Gegengift“ (Plessner 2001a, 281). In seiner Diskussion von Adornos Fragment gebliebener ästhetischer Theorie hebt Plessner deren Wahrheitsorientierung hervor, erneut mit Verweis auf einen nicht dualistisch gelesenen, aber gleichwohl an seinem Agnostizismus festhaltenden Kant:

Wahrheit hat Kunst (deren Medium die Sinnenschönheit ist) als ,Schein des Scheinlosen“. So wird sie in einer Welt, deren Erkenntnisideal nachprüfbare Verifikation nach dem Maße von Falsifikationsmöglichkeit ist, zum Absoluten und die Ästhetik zur Zuflucht der Metaphysik. Ist nach Kantischer Doktrin dem Menschen das Ansich versperrt, ,prägt es in den Kunstwerken, ihrem einheimischen Reich, in dem es keine Differenz zwischen dem Ansich und Für uns geben soll, Rätselfiguren'. (Plessner 2001f, 294f.)

Adorno bleibe, besser als Hegels Idee, durch das Scheinlose dieses Scheins bei der abgebrochenen Transzendenz, d. h. auf der Schwelle der negativen Theologie stehen, was zu Samuel Beckett wohl passe. Gleichwohl hält Plessner die Schwermut in dieser ästhetischen Theorie kaum aus: „Alles Leichte muss sich rechtfertigen, alles Leuchtende hat zu seinem Grunde schwarz. Bezeichnend ist, dass der Begriff des Spiels sich nur an einer Stelle findet, in der von Schiller die Rede ist“ (Plessner 2001f, 295). Bekanntlich versteht Plessner das personale Leben wesentlich als homo ludens, $d$. h. aus dem Spielen in und dem Schauspielen mit soziokulturellen Rollen für Körperleiber von Personen. Dort entsteht die Erfahrung der Negativität nicht allein aus dem Weinen, das von der Welt abschließt, sondern vielmehr aus dem Lachen, das Welt eröffnet, und aus dem Lächeln, das eine souveräne Selbstbegrenzung affirmiert (siehe zweiten Teil im vorliegenden Buch). Plessner vermerkt direkt auch die Bedeutung Walter Benjamins für Adorno: „[D]ie Gesellschaft ist wesentlich die Substanz des Individuums. Das ist doppeldeutig, je nachdem der Nachdruck auf den einzelnen oder das Ganze fällt“ (Plessner 2001e, 335). Die „archaische Rationalität“ im Kunstwerk, die dessen Autonomie in der Erfahrung paradox werden lässt, erinnert an „die verdrängte Mimesis-Bannung des Übermächtigen durch Anver- 
wandlung und Anähnelung, die mit Nachahmung eines Vorgegebenen nicht zu verwechseln ist“ (Plessner 2001f, 292).

Am Ende seiner Besprechung von Adornos Negativer Dialektik kommt Plessner auf Horkheimers Wiederabdruck seiner alten Bemerkungen zur philosophischen Anthropologie (hier Horkheimer 1988a) Schelers aus dem Jahre 1935 zurück. Plessner kritisiert die noch 1970 andauernde Ignoranz gegenüber dessen Werken. Horkheimers falsche Behauptung, die moderne Philosophische Anthropologie entspringe demselben Bedürfnis wie die idealistische Philosophie, nämlich durch absolute Prinzipien das Handeln zu rechtfertigen, wird strikt von Plessner zurückgewiesen; ebenso Horkheimers Vorurteil, es ginge in der Philosophischen Anthropologie um eine biologische Grundstruktur, die die Monopole der Tierart Mensch aus sich hervorgehen lasse und eine historische Invarianz präjudiziere. Das Gegenteil sei der Fall: „Die gesuchte Struktur ermöglicht gerade die ethnologisch bekannte extreme Variabilität des Menschenmöglichen. Sie bildet sogar das Bindeglied zu einem ,historischen Materialismus““, sofern er sich nicht auf überzeitliche Maßstäbe theologischer Deutungen von Geschichte berufe (Plessner 2001a, 278). Horkheimers Manöver, die Philosophische Anthropologie kritisieren zu können, ohne sie zur Kenntnis genommen zu haben, lenke von der Frage nach dem Zusammenhang zwischen der Naturgeschichte und der Gesellschaftsgeschichte ab.

\subsection{Habermas' Anthropologie-Artikel (1958): Die neue Forschungsstrategie der Komplementarität}

Mit Habermas ergreift 1958 nicht nur ein Vertreter der neuen Generation das Wort, sondern ein Kenner beider philosophischer Richtungen, in dem sich die mögliche Rolle eines paradigmatisch Dritten ankündigt (Yos 2019). Er versteht in seinem Anthropologie-Artikel die moderne Philosophische Anthropologie, wie sie von Scheler und Plessner begründet worden war, nicht als eine Ausgliederung eines ursprünglich philosophischen Projekts aus der Philosophie in die empirischen Wissenschaften, sondern umgekehrt als eine Reaktion der Philosophie auf die empirischen Wissenschaften vom Menschen, um ihnen ihren Gegenstand und Anspruch erneut philosophisch streitig zu machen (siehe Habermas 1958, 20). Beim Wesen des Menschen handle es sich nämlich um einen Gegenstand, der nicht geradewegs wie ein „[E]twas“ zum „Gegenstand“ gemacht werden könne (ebd., 18). „Über das, wie es sich“ mit dem Menschen „verhält, erfahren wir ernsthaft nur in dem Maße, in dem wir wissen, wer er ist“ (ebd.). Unter Verweis auf Plessners hermeneutische Dimension hebt Habermas hervor, dass sich im Falle des Menschen Sein und Sinn nicht trennen lassen, 
sondern in den Vermögen, auf die sich der Mensch versteht und zu denen er sich versteht, zusammenfallen. Habermas unterscheidet zwischen Schelers seinsmetaphysischer, Plessners von Metaphysik befreiender und Gehlens empirischer Anthropologie (ebd., 24, 26). Plessner löse die Philosophische Anthropologie aus der metaphysischen Klammer, indem es in ihr nicht mehr um Prinzipien oder Substanzen, sondern um Strukturen im Verhältnis des Leibes zur Umwelt gehe, seine Anthropologie sich methodisch neutral gegenüber dem Dualismus (entweder physisch oder psychisch) verhalte und die Gleichursprünglichkeit des Körperlichen und Geistigen im menschlichen Verhalten, der Sprache, der Gestaltung und der Gebärde aufzeige. Die Exzentrierung der Positionalität führe in einen wirklichen Bruch der menschlichen Natur hinein, weil sie nicht die Zentrierung der Positionalität als solche überwinden könne. Es entstehe so der menschlichen Lebensführung das Problem, zwischen Leibsein und Körperhaben ausgleichen zu müssen, weshalb der Mensch von Natur aus künstlich sei (ebd., 25).

Außer Plessners Stufen des Organischen und der Mensch (1928, hier 1975) wird auch sein Buch Lachen und Weinen (1941, hier 1982f) über Ausdrucksgebärden personaler Verhaltensgrenzen besprochen sowie auf seine Ästhesiologie des Geistes in der Einheit der Sinne (1923, hier 1980a) verwiesen. Plessners Macht und menschliche Natur (1931, hier 1981d) und Das Schicksal deutschen Geistes (1935, hier 1974), d. h. Plessners politische Anthropologie im Zeichen der geschichtsphilosophischen Unergründlichkeit des Menschen und die dazu passende geschichtsphilosophische Fallstudie zum Vergleich der deutschen mit der westeuropäischen Entwicklung fehlen. Ebenso unberücksichtigt bleibt Plessners Buch Grenzen der Gemeinschaft. Zur Kritik des sozialen Radikalismus (1924, hier 1981b), d. h. sein sozialphilosophischer Einsatz. Daher entsteht in Habermas’ Artikel der Eindruck, dass Plessner das Problem der „Verschränkung von Umweltbindung und Weltoffenheit“ im personalen Leben nicht richtig stellen könne, wofür man daher Erich Rothackers Kulturanthropologie der sozialen Lebensstile brauche (ebd., 31). ${ }^{7}$ Vor allem aber entsteht der Eindruck, dass die Philosophische Anthropologie, selbst nicht die von Plessner, nicht von sich aus kritisch verfahren könne, sondern dafür das „Wechselgespräch mit einer Theo-

7 Plessner bestand auch gegen Rothacker kategorial darauf, eine biosoziale Umwelt, die nicht durch Weltoffenheit ermöglicht wird, nicht mit einer soziokulturellen Umwelt zu verwechseln, die durch Weltoffenheit ermöglicht wird, letztere aber nur fragmentarisch realisieren kann. Zudem rechnete er mit der „Möglichkeit, dass beim Menschen Umweltgebundenheit und Weltoffenheit kollidieren und nur im Verhältnis einer nicht zum Ausgleich zu bringenden gegenseitigen Verschränkung gelten“ (Plessner 1983k, 80, Hervorh. im Orig.). Vgl. ebenso ders. 1983e, 180-189. 
rie der Gesellschaft“ benötige (Habermas 1958, 34). Der interessante Punkt besteht hier nicht nur darin, dass Habermas Plessners Philosophische Anthropologie halbiert, d.h. unter Absehung ihrer sozial- und geschichtsphilosophischen Fundierung allein ihre Naturphilosophie und Ausdruckstheorie übrig lässt (vgl. dagegen das nicht nur „vertikale“, sondern auch „horizontale“ Verfahren in Plessner 1975, 32, 36). Vielmehr entwirft Habermas dadurch eine forschungsstrategische Komplementarität, nämlich die kritische Verbindung von philosophischer Anthropologie und kritischer Theorie der Gesellschaft, die er historisch unter Verweis auf Marx und aktuell auf Horkheimer, Adorno und Herbert Marcuse für möglich hält. Empirisch passe dazu Gehlens Selbstkorrektur, vom instrumentellen Handeln zum darstellenden Verhalten im Ritus überzugehen, was in Horkheimers und Adornos Dialektik der Aufklärung der Mimesis-Konzeption entspreche (Habermas 1958, 28f). Allerdings weist Habermas Gehlens Versuch, archaische Institutionen zum Maßstab für moderne Gesellschaften zu erklären, kategorisch als „,autoritär“ (ebd., 33) ab. ${ }^{8}$

Es gibt auch einige Anzeichen dafür, dass sich Habermas an seine eigene Forschungsstrategie der Komplementarität bis $\mathrm{zu}$ Erkenntnis und Interesse (1968) gehalten hat. So verweist er zustimmend in seinem Strukturwandel der Öffentlichkeit (1962) auf Plessners andere Hälfte, d. h. auf die Grenzen der Gemeinschaft (1924, hier 1981b), die Verspätete Nation (1959, hier 1974) und den oben erwähnten Aufsatz Das Problem der Öffentlichkeit und die Idee der Entfremdung (1960, hier 1985b), ohne Plessners Konzeption im Ganzen zu übernehmen. Die neomarxistische und hermeneutisch-historische Konzeption von Habermas gehört aber u. a. durch ihre Bezüge auf Plessners und Hannah Arendts Konzeptionen der Öffentlichkeit im Unterschied zum Privaten zur paradigmatischen Wende zu einer öffentlich anspruchsvollen Demokratie. Hier führt Habermas einen neuen, explizit normativen und universalisierbaren Maßstab in die Kritische Theorie ein, an dessen Explikation er ein Leben lang gearbeitet und der sich gegen viele Widerstände mehr und mehr in der Generationenfolge der Kritischen Theorie durchgesetzt hat.

\footnotetext{
8 Fischer stellt Habermas' differenzierte Kritik an Gehlen so dar, als ob es Habermas um „die Selbstauflösung der Philosophischen Anthropologie in eine ,Theorie der Gesellschaft““ gegangen sei. Diese Fehlinterpretation kommt dadurch zustande, dass Fischer selbst die „Philosophische Anthropologie als Denkrichtung“ im „Kern mit Gehlens Werk identifiziert“, wovon sich in Habermas' Text nichts findet (Fischer 2008, 318).
} 


\subsection{Habermas' Kritiken an Plessners Philosophischer Anthropologie von 1972 und 1985}

$\mathrm{Zu}$ Beginn der 1970er Jahre, im Gefolge der 68er-Bewegung, ist Habermas nicht mehr nur davon überzeugt, dass er durch den verständigungsorientierten Sprachgebrauch das erkenntnistheoretische Problem der Wahrheit, d. h. der gegenstandsbezogenen Sprachverwendung, durch eine Konsenstheorie lösen kann (wovon er bekanntlich später abgerückt ist). Er glaubt damals auch, durch die Idealisierung der Sprachverwendungen in intersubjektiver Richtung Max Webers Problem einer Theorie der gesellschaftlichen Rationalisierung lösen zu können. In dieser euphorischen Phase von Habermas' eigener Werkentwicklung - man könnte von einer idealen Hochzeit zwischen Gadamers Sprachhermeneutik und Webers Typenlehre sprechen - nabelt sich Habermas auch von Plessner ab, so in seinem offenen Brief an diesen von 1972. Einerseits würdigt Habermas Plessners „philosophische Rehabilitierung der Natur“: „Eine nichtempiristische, gleichsam Feuerbachsche Parteinahme für das Sinnliche, Widerständige, Äußere, das gleichwohl mit der Sphäre des Sinns, des immer schon Zugänglichen, Inwendigen verwoben ist, verbindet Ihre Position mit der des Marxschen Materialismus“ (Habermas 1981c, 138). Andererseits bemängelt Habermas Plessners Auflösung der „Universalgeschichte in antirevolutionistischer Perspektive“, seinen „nur“ historischen Begriff von Geschichte und Kultur: „Woher nehmen Sie, lieber Herr Plessner, die Sicherheit, dass ein Bildungsprozess der Gattung nicht stattfindet“ (ebd., 140)? Habermas würdigt auch Plessners geniale Deutung von Lachen und Weinen, allerdings unterstellten diese Phänomene eine propositional ausdifferenzierte Sprache. „Intersubjektivität leiten Sie nun nicht aus der Sprache, sondern aus der exzentrischen Position der menschlichen Natur ab“ (ebd.). Habermas formuliert seine Gegenposition in der Frage: Wäre es nicht plausibler, die Struktur des Spiegel-Ichs

unmittelbar aus der Struktur der sprachlichen Kommunikation abzuleiten - und die Bildung der Ich-Identität aus dem Erwerb der Sprachkompetenz, insbesondere aus der Einübung in das System der Personalpronomina? Dann würde sich in dem Doppelaspekt von Leib und Körper die Doppelstruktur der Sprache bloß abbilden (ebd.). ${ }^{9}$

Im Philosophischen Diskurs der Moderne (1985) von Habermas gibt es nur noch die polemische, weil nicht mehr begründete Bemerkung, dass auch die phäno-

9 Honneth/Joas (1980 75-83) haben überzeugend Habermas' sprachidealistische Kritik an Plessner widerlegt, indem sie den Zusammenhang der verschiedenen Expressivitätsformen bei Plessner herausgestellt haben. 
menologische Anthropologie von Plessner wie viele andere ebenso der dichotomisch verfahrenden Subjekt-Philosophie verhaftet bleibe (Habermas 1985, 369). Habermas glaubte damals bekanntlich, durch seine Theorie des kommunikativen Handelns (1981) alle Subjekt-Philosophien durch sprachliche Intersubjektivität überwunden zu haben.

Plessners Programm kann tatsächlich nicht dem theoretischen Ableitungsanspruch von Habermas Genüge leisten. Seine phänomenologische Methode (ohne Husserls dualistische Theorie) dient der Entdeckung von „ursprünglichen“ Phänomenen, die - wie das ungespielte Lachen und das ungespielte Weinen - als Grenzerfahrungen in der personalen Lebensführung unersetzbar sind. Ihre qualitative Irreduzibilität lässt sich nicht aus der Erkenntnis eines anderen Phänomens ,ableiten“. Plessners Hermeneutik erkundet die interkulturelle Vielfalt der Deutungsmöglichkeiten eines solchen Phänomens. Auf dieses kann im Verhalten (im weitesten Sinne) durch Ausdruck, Handlung, Sprache, Gestalten, Prozedur oder Kontaktvermeidung geantwortet werden. Damit der Rückschluss aus einem sprachlichen Verständnis eines nicht allein sprachlichen Phänomens keinen sprachidealistischen Fehlschluss ergibt, müssen ontisch-ontologische Kriterien dafür erfüllt werden, dass das Phänomen nicht nur „angeschaut“ wurde, sondern auch „wirklich“ (Plessner 1975, 114-116, 122) ist. Schließlich sucht Plessners Dialektik nicht nach einem „Generalnenner“ (ebd., 152), aus dem man mehr oder minder alles ableiten könnte, sondern angesichts der Antinomien der reinen Vernunft nach einer solchen Verwirklichung von Skepsis, die die Rückführung der Konstitution auf nichts anderes als ein Subjekt überwindet. Plessners Freilegung von Lebens-,,Sphären“, in denen „Lebenssubjekt und Gegenwelt“ (ebd., 66f) Einheiten der „Eingespieltheit“ (ebd., 202) bilden, während ihr Widerspruch prozediert, bietet gerade einen Ausweg aus der Subjekt-Philosophie. Zudem scheint mir, dass Habermas selbst in seinem Spätwerk die Kritiken von 1972/85 zurücknimmt.

\subsection{Habermas' Rückgriff auf Plessners Körper-Leib-Differenz in Die Zukunft der menschlichen Natur (2001)}

Angesichts des Aufschwungs der biomedizinischen Lebenswissenschaften und insbesondere der Praktiken einer liberalen Eugenik wird Habermas deutlich, dass seine kritische Gesellschaftstheorie keinen Zugang zu den Körperleibern hat, um die es in all den neuen Therapien und Enhancements geht. Seine kommunikativen Aktoren, die sich in ihren kommunikativen Akten um einen verständigungsorientierten Sprachgebrauch zur Koordinierung ihrer Handlungen bemühen, gewinnen durch Rückgriff auf Plessners Differenzierung ein „Leib- 
sein“ und „Körperhaben“ (Habermas 2001, 27f., 64f., 89f.) für Personen in Relation $\mathrm{zu}$ anderen Personen. In der von Habermas angenommenen „Komplementarität“ (Habermas 1981b, 198) zwischen der Lebenswelt (als dem Hintergrund möglicher Kontexte) und der sprachlichen Kommunikation (im aktualen Vordergrund) fehlte das differentielle Leibes-Medium, das die Personen lebensgeschichtlich situiert. Die Lebenswelt kann doch nicht nur aus symbolischen Verweisungshorizonten bestehen, die als lebensweltliche Voraussetzung der Kommunikation aktual im Ganzen opak bleiben, aber im Resultat der verständigungsorientierten Kommunikation $\mathrm{zu}$ den symbolischen Strukturen von Lebenswelt in Kultur (als Wissensvorrat), Gesellschaft (als legitime Ordnung) und Persönlichkeit (als sprach- und handlungsfähiges Subjekt) ausdifferenzieren (ebd., 209). Für die personale Lebensführung müsste wohl die Lebenswelt als eine solche Ermöglichung von Relationen zwischen Personen thematisiert werden, die je in der Differenz zwischen Leibsein und Körperhaben leben, also durch diese Differenz überhaupt erst zum Leben erwachen.

Plessners kategorialer Vorschlag zur Personalität von Menschen (oder anderen Lebewesen) besteht in der Tat aus dieser doppelten Triade, einerseits der Person in ihrer Differenz zwischen Leibsein und Körperhaben, andererseits in den rollenvermittelten Relationen zwischen Personen in Gemeinschafts- und Gesellschaftsformen. Habermas sieht sich in seinem Spätwerk einer Problemlage ausgesetzt, die Plessner 1935 das Problem der neuen „Lebensmächte“ im „Hochkapitalismus“ genannt hatte und die nicht von der Kritischen Theorie aufgegriffen worden ist, obgleich diese sich wohl als dafür verantwortlich hätte begreifen müssen. Plessner verstand unter einer „Lebensmacht“ exemplarisch im Hochkapitalismus Folgendes: Die Ersetzung des lebensweltlichen Krankheitsverständnisses durch eine naturwissenschaftliche Krankheitsdefinition fällt zusammen mit der Erzeugung neuer globaler Märkte der Pharmaindustrie und Einrichtungen der sozialen Hygiene (Plessner 1974, 86-91). Dieses Problem ist, unabhängig von Plessner, leider erst durch Foucaults Konzeption von Biomächten vierzig Jahre später ernst genommen worden.

\subsection{Kritische Anthropologie?}

Ich danke Herbert Schnädelbach für das anregende Stichwort von einer „Kritischen Anthropologie“10. Die Frage nach ihr könnte zu einer Diskussion zwi-

10 Herbert Schnädelbach hat diesen Ausdruck an dem Roundtable der Tagung Mensch und Gesellschaft zwischen Natur und Geschichte: Zum Verhältnis von Philosophischer Anthropologie 
schen Philosophischer Anthropologie und Kritischer Theorie einladen, wie sie von Habermas begonnen und von Honneth und Joas (1980) erneuert worden ist. Ich bleibe hier bei meiner kleinen Auswahl aus diesem großen Thema und beziehe sie auf künftige Aufgaben aus meiner Sicht der Philosophischen Anthropologie Plessners.

Erstens: Das in diesem Vergleich auffälligste Defizit der Kritischen Theorie besteht in dem Fehlen einer Philosophie der unbelebten und belebten Natur, darunter der pflanzlichen, tierlichen und personalen Lebensformen, von denen die letzteren nicht ohne die ersteren als Sphäre existieren können. Dieser $\mathrm{Zu}-$ sammenhang ist inzwischen als die ökologische Frage bekannt und zu Recht auf der politischen Agenda. Plessners Naturphilosophie bietet dafür zwar ein besseres Potential als nur die sozial-kritische Herausstellung des Zusammenhangs zwischen der Beherrschung von Menschen und der Beherrschung der äußeren Natur, aber auch seine Naturphilosophie ist bisher nicht ökologisch expliziert worden. Die ökologische Erweiterung des Verständnisses von Lebenswelt auf ihre nachhaltigen biologischen Reproduktionsvoraussetzungen steht längst an.

Zweitens: Was die Frage des Verhältnisses zur eigenen Natur personaler Lebewesen betrifft, hat Plessner zwar das Problem neuer „Lebensmächte“ im Hochkapitalismus entdeckt, es aber nicht konzeptionell ausgeführt (siehe Krüger 2009a). Strukturell und funktional bietet sein Ansatz einen Zugang zum Problem, indem man der Triangulation seiner beiden Triaden zur Personalität des Lebens folgt, also Personalität sowohl im Hinblick auf die Körper-LeibDifferenz als auch im Hinblick auf die interpersonalen Relationen in Gemeinschafts- und Gesellschaftsformen aufschlüsselt. Der kritische Zugang zur eigenen Natur über die Psychoanalyse ist in Plessners Konzeption insofern vorgesehen, als er nicht gesellschaftlich dazu führt, über die „Utopie der verlorenen Wildform“ dann am Ende doch in der „biologischen Deutung der Zivilisation als eines Sündenfalls der Natur“ (Plessner 1983e, 193; siehe im vorliegenden Buch 6. Kap.) zu landen. Seine Bejahung der Übernahme von Verantwortung in funktional produktiven Machtbeziehungen begnügt sich nicht mit der Kritik an der Herrschaft der Anderen im Namen der eigenen Emanzipation von Herrschaft (siehe Plessners grundlegenden Aufsatz Die Emanzipation der Macht (1962, hier 1981a; Krüger 2009a u. 10).

Drittens: Versteht man die Personalität des Lebens im genannten Sinne, erweitert sich auch das Kommunikationsverständnis auf die Responsivität

und Kritischer Theorie am 12. 2. 2016 an der Universität Potsdam (in Kooperation mit dem Institut für Philosophie der Freien Universität Berlin) vorgeschlagen. 
für alle möglichen Formen von Expressivität, Mimesis eingeschlossen. Für Plessners öffentliche Untersuchungsart war seit seiner Ästhesiologie des Geistes (1923) die wichtigste Symbolfunktion die, zwischen der aisthetischen „Thematisierung“, der sprachlichen „Präzisierung“ und der „Schemabildung“ (Plessner 1980b, 87, 154, 276; siehe auch Plessner 1980a, 352f.) (in Technik und Wissenschaft) einen Zusammenhang des Fragens und des Antwortens herzustellen, um aus dem falschen Gegensatz von entweder sprachlichem oder nichtsprachlichem Verhalten herauszuführen. So erweitert kann man Habermas' gesellschaftstheoretischem Kreismodell, die gesellschaftlich öffentliche Kommunikation habe die Lebenswelt nicht nur zur Voraussetzung, sondern auch geschichtlich zum Resultat, als Rahmenorientierung folgen. Was diese Rahmenorientierung auf diese Weise an dem Rationalitätsideal einer sachlichen Argumentationsgemeinschaft einbüßt, gewinnt sie an dem Kategorischen Konjunktiv erweiterter Kommunikation mit größeren Partizipationschancen in der Generationenfolge von Habitualisierungsprozessen. Was auch ein derart leiblich erweitertes Kommunikationsverständnis (Rosa 2016) nicht aus eigener Kraft $\mathrm{zu}$ attrahieren vermag, führt doch $\mathrm{zu}$ ihm hin, weil aus den Erfahrungen der gewaltsamen Zerstörung von Körper-Leib-Differenzen für Personen heraus (Bernstein 2015).

Viertens: Habermas' und Plessners Paradigmen überlappen sich im Primat einer öffentlich anspruchsvollen Demokratie, die die ihr funktionalen Wirtschafts- und Verwaltungsformen erkämpfen kann. Adornos „negative Dialektik“ und Plessners „negative Anthropologie“ (Gamm) überschneiden sich in einer Eröffnung von Zukunft wider die Festschreibung bisheriger Positivitäten zu Determinationszwängen. Über die Herausbildung einer „pluralistischen Gesellschaft“, über deren Konflikte man sich keine Illusionen machen möge, „d. h. einer Gesellschaft offen miteinander konkurrierender Wertesysteme“ (Plessner 1983m, 128), schreibt Plessner:

\footnotetext{
$\mathrm{Zu}$ dieser unabgeschlossenen Transformation in einer Gesellschaft ohne vorgegebene $\mathrm{Au}$ torität, d. h. der vollendeten Aufklärung, passt die Scheu vor der Fixierung menschlichen Wesens und seiner Bestimmung in einem nicht mehr revidierbaren Sinne. Was vom Standpunkt autoritäts- und traditionsgebundener Weltordnung und Gesellschaftsverfassung sich als Schwäche, Unentschlossenheit, Ratlosigkeit und Angst ausnimmt, erhält im Aspekt der werdenden Gesellschaftsordnung den Wert der Stärke und des Mutes zur Freiheit. (Ebd.; siehe auch ebd., 134)
}

Fünftens: Schließlich und zuvörderst geht es um die Spezifik der beiden Arten und Weisen zu philosophieren, die transdisziplinäre Zusammenarbeit erst ermöglicht. Plessner forderte die Distanzierung von der anthropozentrischen Spielregel modernen Philosophierens, sich gegenseitig durch Entweder-oder- 
Alternativen in einer Serie kopernikanischer Revolutionen aushebeln zu wollen, was längst dem Schema kapitalistisch verwertbarer Konkurrenz entspricht. Er sah den der Philosophie spezifischen Werthorizont in der Bewahrung der Würde endlicher Vernunftwesen in ihrer Lebensführung (Plessner 1981e, 270-277). Die Idee der Würde schützt das Individuum davor, entweder dieser Gemeinschaftsform oder jener Gesellschaftsform geopfert werden zu dürfen oder gar zu müssen. Umgekehrt: Jede individuelle Person braucht sowohl Gemeinschaftsals auch Gesellschaftsformen, denn sie unterliegt in ihrem Werdensprozess einer „ontisch-ontologischen Zweideutigkeit“ (Plessner 1981b, 63-69, 79-81, 91-94). „In uns selbst liegen neben den gemeinschaftsverlangenden und gemeinschaftsstützenden die gesellschaftsverlangenden, distanzierenden Mächte des Leibes nicht weniger wie der Seele, in jeder Sozialbeziehung wartet die eine, wenn noch die andere gilt, auf ihre Erweckung“ (ebd., 115). ${ }^{11}$ Für beide philosophische Richtungen sind einerseits verschiedene Kant-, Hegel- und Marx-Transformationen tragend, andererseits verschiedene systematische wie essayistische Kombinationen aus phänomenologischem, hermeneutischem und dialektischem Vorgehen durch Negativität, d. h. ohne absolute Ansprüche auf eine positive Identität im Ganzen. Habermas hat 1958 völlig zu Recht die Verschränkung von Umwelt und Welt als systematisches Kernproblem hervorgehoben. Wie wird die fragmentarische Eröffnung von Welt philosophisch ermöglicht, ohne erneut im gnoseologisch-ontologischen Selbstprivileg eines individuellen oder kollektiven Selbstbewusstseins hängen zu bleiben?

11 Plessner denkt das Verhältnis Individuum - Gemeinschaft - Gesellschaft unter dem Primat der Würde des Einzelnen, d. h. aus dem „Schicksal der Individualisierung“ (Plessner 1981b, 60) heraus, das keinem Individuum von einer Sozialbeziehung abgenommen werden kann. Dieses Primat bedeutet aber keinen „Solipsismus“, so Honneth/Joas (1980, 83-84), sondern eine wertedemokratische, $d . h$. nicht der Mehrheitsregel zu unterwerfende Grenze für Vergemeinschaftungs- und Vergesellschaftungsprozesse von Individuen. Plessner ist, aus der negativen Erfahrung des Weimarer Bürgerkriegs heraus, ein Vordenker des Grundgesetzes, Artikel 1, Absatz 1. 